\title{
Proximal Gastrectomy with Gastric Tube Reconstruction or Jejunal Interposition Reconstruction in the Upper Third Gastric Cancer: Which is Better for the Surgical Short-Term Outcomes.
}

\section{Zhiguo Li}

Harbin Medical University Third Clinical College: Tumor Hospital of Harbin Medical University

\section{Yan Ma}

Harbin Medical University Third Clinical College: Tumor Hospital of Harbin Medical University

\section{Guiting Liu}

Harbin Medical University Third Clinical College: Tumor Hospital of Harbin Medical University

\section{Ming Fang}

Harbin Medical University Third Clinical College: Tumor Hospital of Harbin Medical University

YingWei Xue ( $\sim$ xueyingwei@hrbmu.edu.cn)

Harbin Medical University Third Hospital: Tumor Hospital of Harbin Medical University

\section{Research article}

Keywords: ejunal interposition (JI), gastric tube (GT), Gastrointestinal Symptom Rating Scale (GSRS), Los Angeles (LA)

Posted Date: February 9th, 2021

DOl: https://doi.org/10.21203/rs.3.rs-194845/v1

License: (c) (i) This work is licensed under a Creative Commons Attribution 4.0 International License. Read Full License

Version of Record: A version of this preprint was published at BMC Surgery on July 5th, 2021. See the published version at https://doi.org/10.1186/s12893-021-01239-7. 


\section{Abstract}

Objective: Proximal gastrectomy was acted as a function-preserving operation for upper third gastric cancer. The aim of this study is to compare the surgical short-term outcomes between proximal gastrectomy with gastric tube reconstruction and proximal gastrectomy with Jejunal interposition reconstruction in the upper third gastric cancer.

Methods: A retrospective review of 301 patients who underwent proximal gastrectomy with jejunal interposition (JI) or gastric tube (GT) at the Harbin Medical University Cancer Hospital between June 2007 and December 2016 was performed. The Gastrointestinal Symptom Rating Scale (GSRS) and Visick grade were used to evaluate the post-gastrectomy syndromes. The gastrointestinal fiberoscopy was used to evaluate the prevalence and severity of reflux esophagitis by Los Angeles (LA) classification system.

Results: The JI group had a longer operation time than GT group ( $220 \pm 52$ vs $182 \pm 50 \mathrm{~min}$ ), whereas there was no significant difference in blood loss. Compared to the GT group, the Visick grade and GSRS score were significantly higher than that of the JI group. The reflux esophagitis of GT group was significantly higher than that of the JI group.

Conclusion: Proximal gastrectomy is well tolerated, with excellent short-outcomes in patients with upper third gastric cancer . C ompared with GT construction, Jl construction has clear functional advantages and may provide better quality of life for patients with upper third gastric cancer.

\section{Full Text}

Due to technical limitations, full-text HTML conversion of this manuscript could not be completed. However, the latest manuscript can be downloaded and accessed as a PDF.

\section{Tables}

Table 1 Clinicopathological characteristics of patients who underwent JI or GT reconstruction 


\begin{tabular}{|c|c|c|c|c|}
\hline Parameters & Total & Jl group & GT group & $P$ value \\
\hline Cases (n) & 301 & 150 & 151 & \\
\hline Age (years) & & & & 0.032 \\
\hline$\$ 60$ & 136 & 77 & 59 & \\
\hline$\geq 60$ & 165 & 73 & 92 & \\
\hline Gender & & & & 0.791 \\
\hline Male & 243 & 122 & 121 & \\
\hline Female & 58 & 28 & 30 & \\
\hline Height (cm) & $170 \pm 7$ & $171 \pm 6$ & $168 \pm 7$ & $\nabla 0.001^{* *}$ \\
\hline Weight (Kg) & $68 \pm 11$ & $67 \pm 10$ & $68 \pm 12$ & 0.433 \\
\hline BMI & $23.66 \pm 3.75$ & $23.28 \pm 3.95$ & $24.04 \pm 3.50$ & 0.078 \\
\hline Hospital stay (day) & $23 \pm 3$ & $21 \pm 1$ & $25 \pm 5$ & $\nabla 0.001^{\star \star}$ \\
\hline Operating time (min) & $201 \pm 54$ & $220 \pm 52$ & $182 \pm 50$ & $\nabla 0.001^{* *}$ \\
\hline Blood loss (ml) & $162 \pm 95$ & $169 \pm 84$ & $155 \pm 104$ & 0.200 \\
\hline Tumor size (cm) & & & & 0.778 \\
\hline 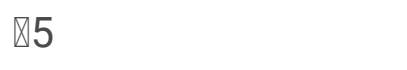 & 179 & 88 & 91 & \\
\hline$\geq 5$ & 122 & 62 & 60 & \\
\hline ABO blood type & & & & 0.745 \\
\hline A & 78 & 44 & 34 & \\
\hline B & 105 & 51 & 54 & \\
\hline 0 & 91 & 42 & 49 & \\
\hline$A B$ & 27 & 13 & 14 & \\
\hline \multicolumn{5}{|c|}{ Pathological TNM classification } \\
\hline T stage & & & & $\nabla 0.001^{* *}$ \\
\hline T1 & 34 & 14 & 20 & \\
\hline T2 & 46 & 21 & 25 & \\
\hline T3 & 96 & 28 & 68 & \\
\hline $\mathrm{T} 4$ & 125 & 87 & 38 & \\
\hline $\mathrm{N}$ stage & & & & $\triangle 0.05^{\star}$ \\
\hline
\end{tabular}




\begin{tabular}{|c|c|c|c|c|}
\hline NO & 141 & 69 & 72 & \\
\hline N1 & 64 & 26 & 38 & \\
\hline N2 & 57 & 38 & 19 & \\
\hline N3 & 39 & 17 & 22 & \\
\hline TNM stage & & & & 0.093 \\
\hline I & 65 & 27 & 38 & \\
\hline II & 119 & 56 & 63 & \\
\hline III & 117 & 67 & 50 & \\
\hline Lauren Classification & & & & 0.144 \\
\hline Diffuse type & 18 & 5 & 13 & \\
\hline Intestinal type & 208 & 108 & 100 & \\
\hline Mixed type & 75 & 37 & 38 & \\
\hline Differentiation & & & & $\otimes 0.05^{\star}$ \\
\hline poorly differentiated & 126 & 73 & 53 & \\
\hline moderately differentiated & 143 & 65 & 78 & \\
\hline well differentiated & 32 & 12 & 20 & \\
\hline Vessel invasion & & & & 0.867 \\
\hline positive & 166 & 82 & 84 & \\
\hline negative & 135 & 68 & 67 & \\
\hline Nerve invasion & & & & $0.001^{\star}$ \\
\hline positive & 204 & 115 & 89 & \\
\hline negative & 97 & 35 & 62 & \\
\hline Pathology & & & & $\varangle 0.001^{\star *}$ \\
\hline adenocarcinoma & 211 & 119 & 92 & \\
\hline mucinous carcinoma & 62 & 15 & 47 & \\
\hline signet ring cell carcinoma & 18 & 12 & 6 & \\
\hline others & 10 & 4 & 6 & \\
\hline Total lymph nodes & & & & $\varangle 0.001^{* \star}$ \\
\hline$\nabla 20$ & 168 & 109 & 59 & \\
\hline
\end{tabular}




\begin{tabular}{|c|c|c|c|c|}
\hline$\geq 20$ & 133 & 41 & 92 & \\
\hline Positive lymph nodes & & & & $\llbracket 0.001^{* *}$ \\
\hline$\varangle 3$ & 154 & 95 & 59 & \\
\hline$\geq 3$ & 147 & 55 & 92 & \\
\hline
\end{tabular}

$\star: P<0.05 ; * \star: P<0.001$

Table 2 Postoperative functional outcomes

\begin{tabular}{|c|c|c|c|c|}
\hline Parameters & Total & JI group & GT group & $P$ value \\
\hline Cases (n) & 301 & 150 & 151 & \\
\hline Visick grade & & & & $<0.05^{\star}$ \\
\hline V1 & 201 & 112 & 89 & \\
\hline V2 & 71 & 29 & 42 & \\
\hline V3 & 19 & 7 & 12 & \\
\hline V4 & 10 & 2 & 8 & \\
\hline GSRS score & $26.89 \pm 5.06$ & $25.58 \pm 4.56$ & $28.48 \pm 5.78$ & 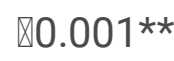 \\
\hline
\end{tabular}

$\star: P<0.05 ; * *: P<0.001$

Table 3 Comparison of preoperative and postoperative nutritional outcomes between groups 


\begin{tabular}{|c|c|c|c|c|}
\hline Parameters & Total & Jl group & GT group & $P$ value \\
\hline Cases (n) & 301 & 150 & 151 & \\
\hline \multicolumn{5}{|l|}{ Preoperative } \\
\hline Total protein $(\mathrm{g} / \mathrm{l})$ & $68.62 \pm 7.92$ & $68.23 \pm 6.37$ & $69.00 \pm 9.20$ & 0.399 \\
\hline Albumin $(\mathrm{g} / \mathrm{l})$ & $42.44 \pm 4.57$ & $43.63 \pm 4.65$ & $41.25 \pm 4.18$ & $\llbracket 0.001^{\star \star}$ \\
\hline Globulin (g/l) & $26.04 \pm 5.02$ & $24.62 \pm 5.38$ & $27.44 \pm 4.20$ & $\llbracket 0.001^{\star \star}$ \\
\hline Prealbumin $(\mathrm{g} / \mathrm{l})$ & $254.68 \pm 66.74$ & $252.91 \pm 67.49$ & $256.44 \pm 66.17$ & 0.647 \\
\hline White blood cell (W) & $6.62 \pm 2.07$ & $6.38 \pm 2.05$ & $6.86 \pm 2.07$ & $0.044^{\star}$ \\
\hline Neutrophils (N) & $3.89 \pm 1.61$ & $3.67 \pm 1.36$ & $4.12 \pm 1.80$ & $0.015^{\star}$ \\
\hline Lymphocyte (L) & $2.01 \pm 0.69$ & $1.98 \pm 0.66$ & $2.04 \pm 0.72$ & 0.452 \\
\hline Monocyte (M) & $0.49 \pm 0.20$ & $0.45 \pm 0.20$ & $0.52 \pm 0.20$ & $0.003^{*}$ \\
\hline Hemoglobin $(\mathrm{Hb})$ & $133.01 \pm 23.44$ & $132.81 \pm 21.71$ & $133.22 \pm 25.11$ & 0.880 \\
\hline Erythrocyte (E) & $4.41 \pm 0.59$ & $4.39 \pm 0.55$ & $4.44 \pm 0.63$ & 0.464 \\
\hline Platelet (P) & $239.54 \pm 75.45$ & $250.79 \pm 82.61$ & $228.36 \pm 65.98$ & $0.010 *$ \\
\hline \multicolumn{5}{|l|}{ Postoperative } \\
\hline Total protein $(\mathrm{g} / \mathrm{l})$ & $54.44 \pm 5.72$ & $53.64 \pm 6.13$ & $55.23 \pm 5.19$ & 0.016 \\
\hline Albumin (g/l) & $32.57 \pm 4.08$ & $33.11 \pm 4.55$ & $32.02 \pm 3.48$ & 0.020 \\
\hline Globulin (g/l) & $21.79 \pm 4.56$ & $20.53 \pm 5.22$ & $23.04 \pm 3.38$ & $\llbracket 0.001^{\star \star}$ \\
\hline Prealbumin (g/l) & $172.06 \pm 44.29$ & $170.63 \pm 44.76$ & $173.49 \pm 43.92$ & 0.576 \\
\hline White blood cell (W) & $11.75 \pm 3.65$ & $11.61 \pm 3.87$ & $11.90 \pm 3.42$ & 0.491 \\
\hline Neutrophils (N) & $8.07 \pm 3.82$ & $5.87 \pm 3.03$ & $10.26 \pm 3.23$ & $\llbracket 0.001^{\star \star}$ \\
\hline Lymphocyte (L) & $0.95 \pm 0.45$ & $1.03 \pm 0.50$ & $0.87 \pm 0.37$ & $0.002^{\star *}$ \\
\hline Monocyte (M) & $0.67 \pm 0.28$ & $0.65 \pm 0.28$ & $0.70 \pm 0.28$ & 0.122 \\
\hline Hemoglobin $(\mathrm{Hb})$ & $122.47 \pm 16.42$ & $122.32 \pm 17.08$ & $122.63 \pm 15.79$ & 0.870 \\
\hline Erythrocyte (E) & $4.07 \pm 0.51$ & $4.07 \pm 0.53$ & $4.07 \pm 0.50$ & 0.866 \\
\hline Platelet (P) & $203.61 \pm 67.51$ & $212.42 \pm 75.00$ & $194.80 \pm 58.00$ & $0.023^{*}$ \\
\hline
\end{tabular}

$*: P<0.05 ; * *: P<0.001$ 
Table 4 Endoscopic findings of the JI and GT group

\begin{tabular}{|c|c|c|c|c|}
\hline Parameters & Total & JI group & GT group & $P$ value \\
\hline Cases (n) & 301 & 150 & 151 & \\
\hline Los Angeles classification & & 31 & 48 & 0.028 \\
\hline$A$ & & 20 & 30 & \\
\hline$B$ & & 8 & 13 & \\
\hline C & & 3 & 5 & \\
\hline D & & 0 & 0 & \\
\hline
\end{tabular}

$\star: P<0.05 ;$ 